\title{
Regional Blocks Benefit Ambulatory Breast Cancer Surgery Patients After Discharge Home
}

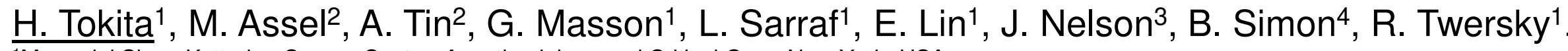

${ }^{1}$ Memorial Sloan Kettering Cancer Center, Anesthesiology and Critical Care, New York, USA.

Memolial Sloan Kettering Cancer Center, Epidemiology and Blostatistics, New York, USA.

${ }^{3}$ Memorial Sloan Kettering Cancer Center, Plastics and Reconstructive Surgery, New York, USA.

${ }^{4}$ Memorial Sloan Kettering Cancer Center, Josie Robertson Surgery Center, New York, USA.

\section{Introduction}

Regional blocks (RB) are increasingly administered to ambulatory mastectomy with immediate reconstruction (MWR) patients but their impact on recovery quality after discharge remains poorly defined. At the Josie Robertson Surgery Center (JRSC), patients are asked to report their symptoms for 10 days after discharge through the MyMSK patient portal and the Recovery Tracker (RT), a 14 question survey adapted from the National Cancer Institute PRO-CTCAE ${ }^{1}$ instrument. We present novel outcome data that correlate the use of $\mathrm{RB}$ with improved post-discharge symptoms in MWR patients.

\section{Methods}

We analyzed data from 713 patients who underwent bilateral MWR from 4/2017 - 12/2018, stratified by patients who received any RB (paravertebral, serratus, and/or PECS blocks) vs. patients who did not. For RTreported pain and fatigue we used generalized estimating equations with a logit link to test association between moderate or greater symptom level and RB receipt, after adjusting for survey day, age, BMI, ASA score and OR time.

\section{Results}

$639(90 \%)$ patients received a RB. Among the 559 patients with at least one RT survey (78\% response rate), the odds of moderate or higher pain was 0.58 times lower among those with a block $(95 \% \mathrm{Cl} 0.34,0.97$; $\mathrm{p}=0.04$; Fig.) with no differences regarding severe pain $(\mathrm{p}=0.7)$. Levels of moderate or higher fatigue also decreased over time, with no significant differences between groups.

\section{Conclusion}

We present novel patient-reported data that suggest RBs benefit MWR patients after discharge. Future studies should include quality and functional outcomes to demonstrate the broader impact of our anesthetic techniques.

\section{References}

1JAMA Oncol 1:1051, 2015.

Figure. Probability of Patient Reporting Moderate or Greater Pain

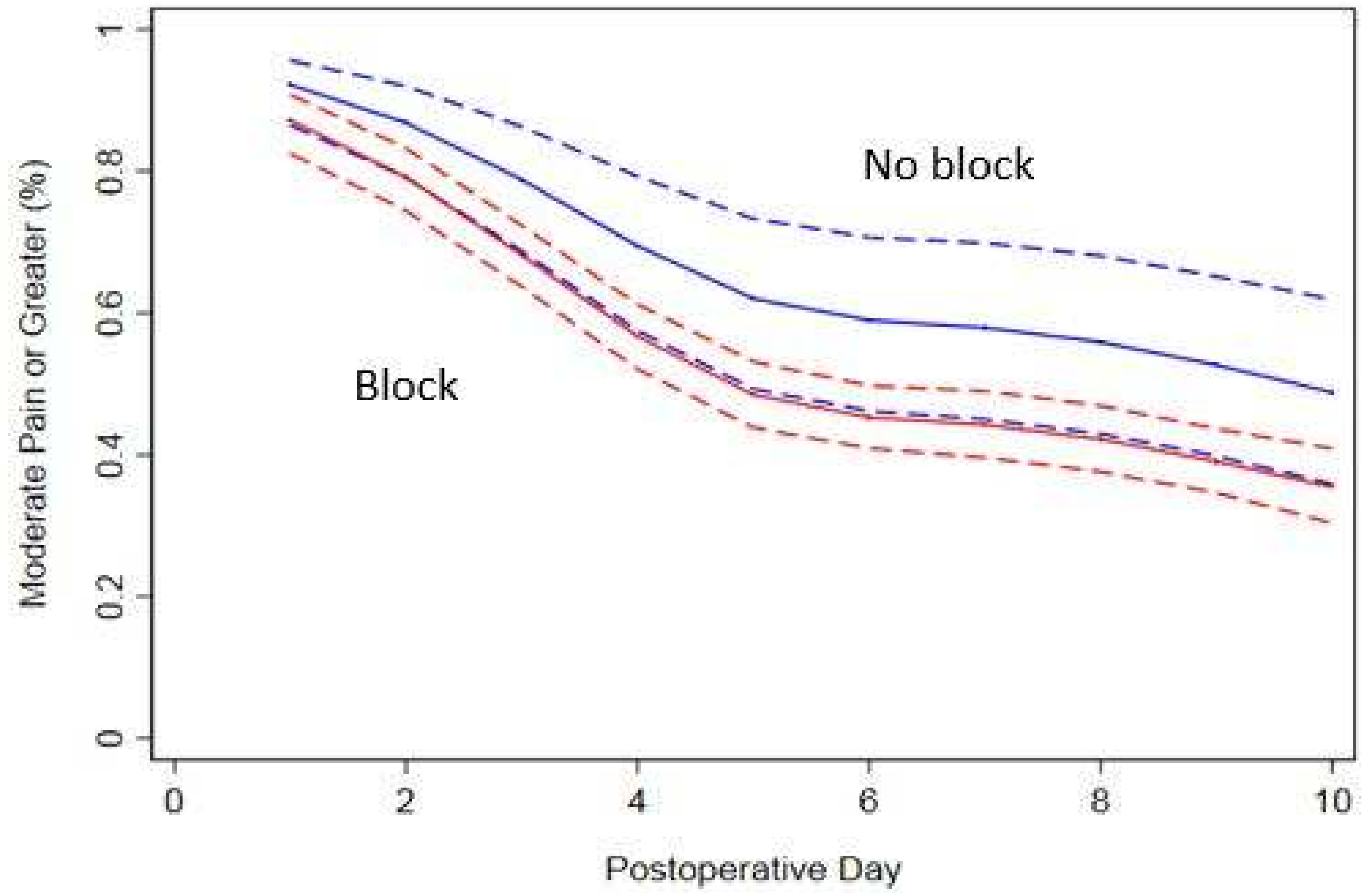

